\title{
Microvascular Coronary Artery Embolism Presenting as an OHCA STEMI Call Detected by CMRI
}

\author{
Napohn Chongprasertpon, Santhosh David \\ Department of Cardiology, Letterkenny University Hospital, Letterkenny, Co. Donegal, Ireland
}

Doi: 10.12890/2020_001772 - European Journal of Case Reports in Internal Medicine - @ EFIM 2020

Received: 02/06/2020

Accepted: 05/06/2020

Published: 29/06/2020

How to cite this article: Chongprasertpon N, David S. Microvascular coronary artery embolism presenting as an OHCA STEMI call detected by CMRI. EJCRIM 2020;7: doi:10.12890/2020_001772.

Conflicts of Interests: The Authors declare that there are no competing interests.

This article is licensed under a Commons Attribution Non-Commercial 4.0 License

\section{ABSTRACT}

We describe a case of coronary artery embolism leading to an out-of-hospital cardiac arrest (OHCA) in which the diagnosis was achieved with utilisation of cardiac magnetic resonance imaging. The patient was otherwise well prior to this episode. Emergency diagnostic coronary angiography revealed patent arteries with TIMI 3 flow. Subsequent cardiac magnetic resonance imaging demonstrated myocardial infarction and focal microvascular obstruction in the infarcted territory. This report describes an uncommon case presentation, highlights areas for improvement in diagnostic criteria, and briefly discusses the currently available data regarding coronary artery embolism.

\section{LEARNING POINTS}

- Coronary artery embolism is uncommon but it is important to accurately diagnose it given the associated poor prognosis.

- Currently proposed scoring systems for the diagnosis of coronary artery embolism do not include the use of cardiac magnetic resonance imaging to identify intra-coronary emboli.

- Consideration should be given to including cardiac magnetic resonance imaging for the detection of intra-coronary emboli as an alternative to angiography in scoring criteria used to diagnose coronary artery embolism.

\section{KEYWORDS}

Cardiac magnetic resonance imaging, coronary artery embolism, ST-segment elevation myocardial infarction

\section{CASE PRESENTATION}

A 75-year-old woman presented to our service having experienced an out-of-hospital cardiac arrest (OHCA) while in transit to our emergency department (ED) via ambulance.

There was no significant background history apart from hypertension treated with olmesartan which was the patient's only medication prior to admission. She had never smoked and does not consume alcohol. She had no family history of note despite being specifically asked about arrythmias, sudden deaths, coronary artery and heart disease as well as thrombophilia.

Two days prior to the presentation leading to the OHCA, the patient developed mild gastrointestinal symptoms of bloating. These symptoms increased in intensity on the day of her OHCA and were associated with epigastric pain but no change in bowel motions or vomiting. Following assessment by her GP, the emergency medical service (EMS) was activated.

The patient developed pulseless ventricular tachycardia (VT) during transit with the EMS. She was promptly treated by paramedics with cardiopulmonary resuscitation, three shocks at $200 \mathrm{~J}$ and two doses of $1 \mathrm{mg}$ of adrenaline according to the American Heart Association Advanced Cardiac Life Support protocol. Subsequently, she attained return of spontaneous circulation. A 12-lead ECG revealed polymorphic ventricular tachycardia (Fig. 1). She had a previously documented normal ECG from a decade earlier and her initial 12-lead ECG in the ambulance, taken 10 minutes prior to her cardiac arrest, did not show any prolongation of the QT interval with a QTc of 428 mS. 


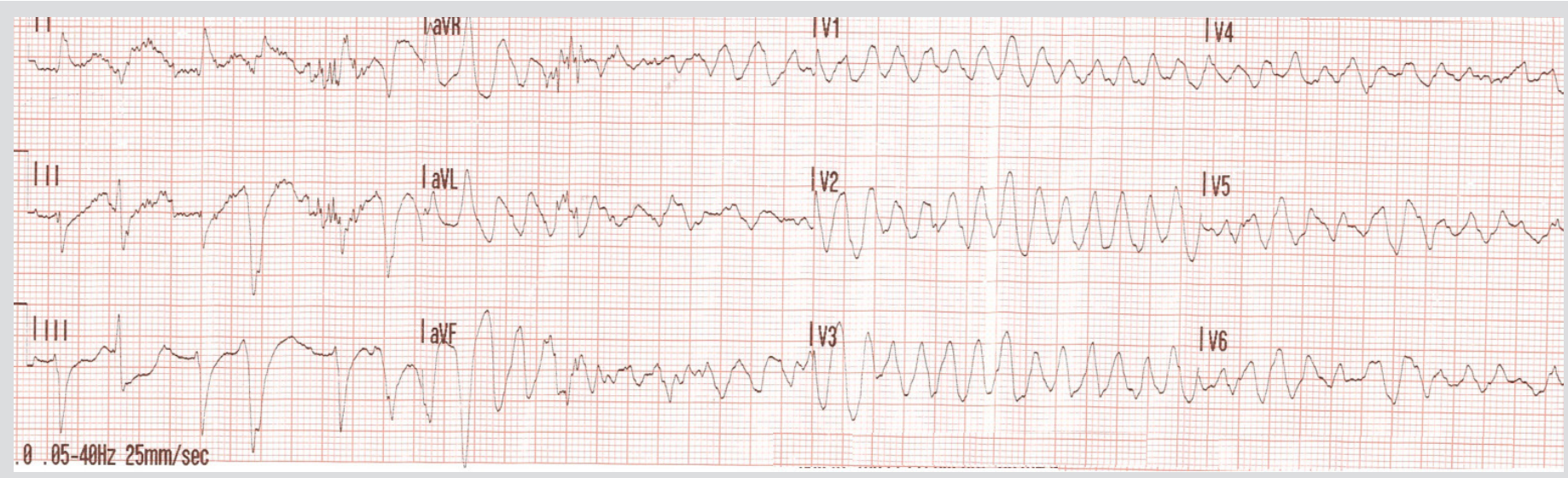

Figure 1. Twelve-lead ECG showing polymorphic ventricular tachycardia

On assessment in the ED, an ECG demonstrated ST-segment elevation in leads II, II and aVF. Therefore, the patient was immediately brought to the cardiac catherization laboratory where a diagnostic coronary angiogram revealed patent coronary arteries with TIMI 3 flow (Fig. 2). The first troponin level prior to her coronary angiogram was $349 \mathrm{ng} / \mathrm{l}$ (0-9 ng/l), which rose the following day to 2,222 ng/l. Her transthoracic echocardiograph showed apical akinesis and septal hypokinesis with an ejection fraction of 30-40\% but a tricuspid annular plane systolic excursion of $24 \mathrm{~mm}$. The main differential diagnosis at this point was myocarditis.

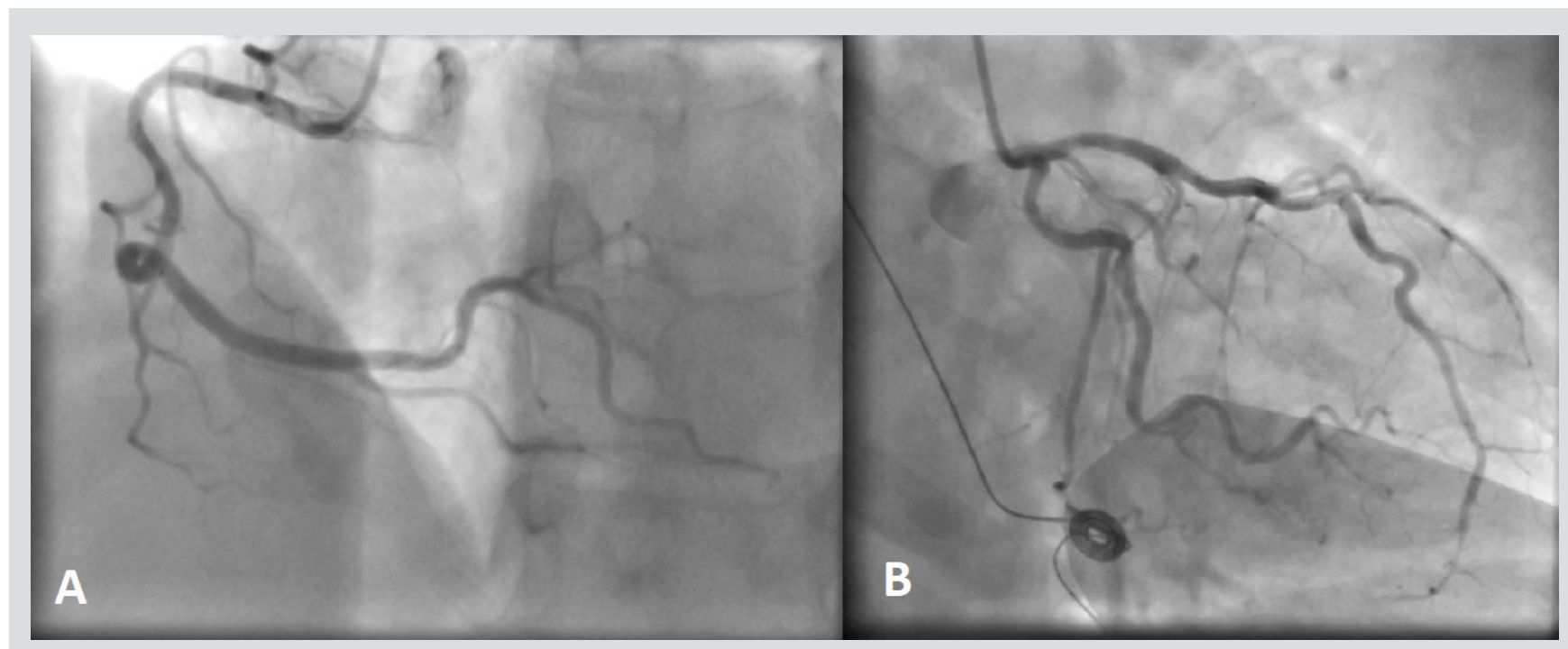

Figure 2. Diagnostic coronary angiogram demonstrating patent coronary arteries with TIMI 3 flow. (A) Right coronary artery; (B) left coronary artery and left circumflex artery

Cardiac magnetic resonance imaging (CMRI) was performed. This was diagnostic of a transmural acute myocardial infarction (AMI) in the distal to apical inferior and inferoseptal wall with persistent myocardial oedema and focal microvascular obstruction in the infarcted territory. This was a case of myocardial infarction with non-obstructive coronary arteries (MINOCA) as per European Society of Cardiology (ESC) diagnostic criteria ${ }^{[1]}$. As there were no concerning features for other underlying aetiologies, we diagnosed a thromboembolic acute myocardial infarction.

There were no episodes of atrial fibrillation during in-patient monitoring in the coronary care unit. Subsequent thrombophilia screening proved negative for anti-nuclear antibody, anti-phospholipid antibody, lupus anticoagulant antibody, neutrophil cytoplasmic antibodies, rheumatoid factor and JAK-2 mutation with a normal haematinic profile and serum homocysteine levels. 
This patient presented during the coronavirus disease 2019 pandemic but repeated nasopharyngeal swabs for severe acute respiratory syndrome coronavirus 2 (SARS-CoV-2) proved negative with negative SARS-CoV-2 serum antibodies. In summary, this is an unusual case of a coronary artery embolism presenting with pulseless polymorphic ventricular tachycardia and diagnosed on CMRI.

\section{DISCUSSION}

Coronary artery embolism is an uncommon phenomenon. A previous study of 1,776 patients demonstrated a coronary embolism prevalence of $2.9 \%$ of cases of new onset acute myocardial infarction ${ }^{[2]}$. Atrial fibrillation was identified as the most common underlying aetiology of coronary artery embolisms at $73 \%$ of cases ${ }^{[2]}$. Coronary artery embolism can be divided into three broad categories, namely, direct, paradoxical and iatrogenic ${ }^{[3]}$. The patient in this case most likely developed a direct coronary artery embolism due to embolic material originating from the left side of the heart or the pulmonary veins.

There remains a paucity of evidence regarding the use of scoring systems for the diagnosis of coronary artery embolism. In 2018 , Raphael et al. proposed a scoring system for diagnosing coronary artery embolism which was adapted from the prior work of Shibata et al. ${ }^{[2,3]}$. The scoring system of Raphael et al. utilises five major and three minor criteria for establishing a diagnosis of probable or definite coronary embolism ${ }^{[3]}$. One of the major criteria in this scoring system is angiographic evidence of coronary embolus or thrombus. The patient in this case did not fulfil the criteria proposed by Raphael et al. ${ }^{[3]}$ for the diagnosis of a probable coronary artery embolism. The patient did not have angiographic evidence of coronary embolus or thrombus and thus did not fulfil any major criteria. However, the CMRI provided evidence of microvascular obstruction in the infarcted territory. This highlights the utility of CMRI in identifying coronary emboli and we would suggest that consideration should be given to incorporating the identification of a coronary obstruction via CMRI into diagnostic scoring systems for coronary artery embolism. Although the scoring criteria for coronary embolism were not fulfilled, this patient with focal microvascular coronary obstruction and consequent myocardial infarction had no evidence of plaque rupture leading to a diagnosis of coronary artery embolism given the lack of evidence for other underlying aetiologies.

As part of the diagnostic work-up of cardiac thromboembolism, the ESC recommends consideration should be given to the use of transoesophageal echocardiography to assess for a patent foramen ovale as well as intravascular ultrasound and optical coherence tomography to evaluate for coronary dissection or thrombosis and atherosclerotic plaque disruption and erosion ${ }^{[1]}$. We did not perform transoesophageal echocardiography for our patient given that this was her first embolic episode at the age of 75 as well as patient preference after discussion of the benefits and risk of the procedure. Intravascular ultrasound or optical coherence tomography were not utilised as the coronary arteries were well visualised during angiography without concerning features.

The prognosis for patients with coronary embolism ST-segment elevation myocardial infarction (STEMI) is worse than for non-coronary embolism STEMI patients, with a 1-year all-cause mortality of $15 \%$ vs $6 \%$, respectively; this was also demonstrated on long-term KaplanMeier analysis ${ }^{[4]}$. Thus, further research to develop and evaluate diagnostic criteria for coronary embolism is important and would be beneficial. We would propose that these include CMRI evidence of coronary artery obstruction as an alternative to angiographic evidence of a coronary embolus as a diagnostic criterion in scoring systems for diagnosing coronary artery embolism.

\section{REFERENCES}

1. Agewall S, Beltrame JF, Reynolds HR, Niessner A, Rosano G, ALP Caforio, et al. ESC working group position paper on myocardial infarction with non-obstructive coronary arteries. Eur Heart J 2017;38(3):143-153.

2. Shibata T, Kawakami S, Noguchi T, Tanaka T, Asaumi Y, Kanaya T, et al. Prevalence, clinical features, and prognosis of acute myocardial infarction due to coronary artery embolism. Circulation 2015;132:241-250.

3. Raphael CE, Heit JA, Reeder GS, Bois MC, Maleszewski JJ, Tilbury RT, et al. Coronary embolus: an underappreciated cause of acute coronary syndromes. JACC Cardiovasc Interv 2018;11(2):172-180.

4. Popovic B, Agrinier N, Bouchahda N, Pinelli S, Maigrat CH, Metzdorf PA. Coronary embolism among ST-segment-elevation myocardial infarction patients: mechanisms and management. Circ Cardiovasc Interv 2018;11(1):e005587. 